\title{
Erratum to: Thermal Expansion Studies of Selected High-Temperature Thermoelectric Materials
}

\author{
VILUPANUR RAVI, ${ }^{1,2,5}$ SAMAD FIRDOSY, ${ }^{1}$ THIERRY CAILLAT, ${ }^{1}$ \\ ERIK BRANDON, ${ }^{1}$ KEITH VAN DER WALDE, ${ }^{3}$ LINA MARICIC, ${ }^{3}$ \\ and ALI SAYIR ${ }^{4}$
}

\begin{abstract}
1.-Jet Propulsion Laboratory, California Institute of Technology, 4800 Oak Grove Drive, Pasadena, CA 91109, USA. 2.-California State Polytechnic University, 3801, W. Temple Avenue, Pomona, CA 91768, USA. 3.-ATA Engineering, Inc., 11995 El Camino Real, San Diego, CA 92130, USA. 4.-NASA Glenn Research Center, 21000 Brookpark Road, Cleveland, OH 44135, USA. 5.—e-mail: varavi@jpl.nasa.gov
\end{abstract}

\section{Erratum to: Journal of ELECTRONIC MATERIALS \\ Vol. 38, No. 7, pp. 1433-1442 \\ DOI 10.1007/s11664-009-0734-2}

1. The corrected thermal linear expansions in Table 1 are as follows:

$p$-nanostructured SiGe

$-1.2933 \times 10^{-10} T^{3}+3.3420 \times 10^{-7} T^{2}+1.8858 \times 10^{-4} T-0.0817(323 \leq T \leq 1273 \mathrm{~K})$

$n$-nanostructured SiGe

$-2.0436 \times 10^{-10} T^{3}+4.6723 \times 10^{-7} T^{2}+1.0680 \times 10^{-4} T-0.0650(323 \leq T \leq 1273 \mathrm{~K})$

$n$-mechanically alloyed SiGe

$-2.2241 \times 10^{-10} T^{3}+5.2298 \times 10^{-7} T^{2}+2.1958 \times 10^{-4} T-0.1125(323 \leq T \leq 1273 \mathrm{~K})$

$p-\mathrm{Yb}_{14} \mathrm{MnSb}_{11}$

$2.5676 \times 10^{-11} T^{3}+4.3797 \times 10^{-8} T^{2}+1.5388 \times 10^{-3} T-0.4735(323 \leq T \leq 1273 \mathrm{~K})$

$n-\mathrm{CoSb}_{3}$

$7.9039 \times 10^{-12} T^{4}-2.1872 \times 10^{-8} T^{3}+2.2211 \times 10^{-5} T^{2}-8.5866 \times 10^{-3} T+1.2422(338 \leq T \leq 974 \mathrm{~K})$

$p-\mathrm{CeFe}_{3} \mathrm{RuSb}_{12}$

$1.4296 \times 10^{-11} T^{4}-3.6799 \times 10^{-8} T^{3}+3.4942 \times 10^{-5} T^{2}-1.3065 \times 10^{-2} T+1.9636(338 \leq T \leq 974 \mathrm{~K})$

2. On page 1435 (left column, toward the bottom), the same correction for the $\Delta L / L_{0}(\%)$ expansion for $p$ - $\mathrm{CeFe}_{3} \mathrm{RuSb}_{12}$ needs to be applied:

$\Delta L / L_{0}=1.4296 \times 10^{-11} T^{4}-3.6799 \times 10^{-8} T^{3}+3.4942 \times 10^{-5} T^{2}-1.3065 \times 10^{-2} T+1.9636$ 